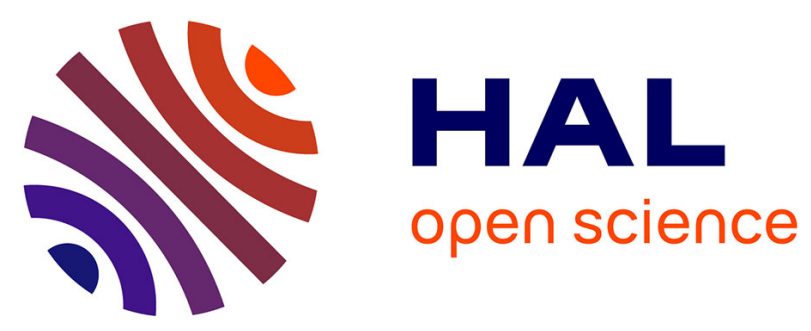

\title{
LE SÉRUM DE CASÉINERIE ET QUELQUES SOUS-PRODUITS DE L'INDUSTRIE LAITIĖRE: LEURS PROPRIÉTÉS ET LEUR VALEUR ALIMENTAIRE
}

Dr Paul Rossi

\section{To cite this version:}

Dr Paul Rossi. LE SÉRUM DE CASÉINERIE ET QUELQUES SOUS-PRODUITS DE L'INDUSTRIE LAITIÈRE: LEURS PROPRIÉTÉS ET LEUR VALEUR ALIMENTAIRE. Le Lait, 1930, 10 (92), pp.136-142. hal-00894985

\section{HAL Id: hal-00894985 \\ https://hal.science/hal-00894985}

Submitted on 1 Jan 1930

HAL is a multi-disciplinary open access archive for the deposit and dissemination of scientific research documents, whether they are published or not. The documents may come from teaching and research institutions in France or abroad, or from public or private research centers.
L'archive ouverte pluridisciplinaire HAL, est destinée au dépôt et à la diffusion de documents scientifiques de niveau recherche, publiés ou non, émanant des établissements d'enseignement et de recherche français ou étrangers, des laboratoires publics ou privés. 


\title{
LE SÉRUIM DE CASÉINERIE ET QUELQUES SOUS-PRODUITS DE L'INDUSTRIE LAITIÈRE: LEURS PROPRIÉTÉS ET LEUR VALEUR ALIMENTAIRE
}

\author{
Par le Dr Paul ROSSI \\ Direteur Régional des Services Vétérinaires de Saône-et-Loire
}

A la suite de la destruction du vignoble par le phylloxera, le cultivateur charentais se lança dans la production laitière et créa, à partir de 1888 , de nombreuses beurreries coopératives.

Après la création à Surgères, en 1904, de la première caséinerie par Kirchner, de Prague, les caséineries se multiplièrent dans l'Ouest. L'extension inattendue de cette industrie devait fournir, à l'elevage porcin, un aliment de haute valeur : Le "sérum " (a), dont il nous a paru intéressant de faire une rapide étude.

Cet aliment est, en effet, trop méconnu et sa valeur alimentaire trop discréditée. Nous passerons en revue sa préparation, ses propriétés physiques et chimiques; puis nous essaierons de dégager le rôle qu'il peut jouer dans l'alimentation et la nutrition du pore; enfin, nous inaiquerons des transformations industrielles, peu connues encore, et nous terminerons en mentionnant quelques autres dérivés de l'industrie du beurre et de la caséine, dont la valeur est loin d'être négligea ble.

Préparation. - A son arrivée à la coopérative, le lait passe dans les écrémeuses centrifuges (b), puis, par des tuyauteries métalliques, est amené dans la caséinerie où il emplit une série de cuves à double paroi. On Je réchauffe à $35^{\circ}$ et on lui ajoute de la présure qu'on mélangera intimement au moyen d'un agitateur de bois. La coagulation se produit en 20 minutes. On met en marche l'agitateur, à la vitesse de 30 tours à la minute, pour briser le "caillé ") et on ouvre la vanne de vapeur de façon à le "cuire "; on chauffe jusqu'à 60-63. L'opération doit durer une demi-heure environ et l'élévation de température doit être régu. lière, c'est-à-dire qu'on doit chercher à ce qu'elle s'élève d'environ un degré par minute. "Quand le caillé est cuit, on ferme la vanne de vapeur et on place, sous la bonde de la cuve, un tamis métallique ou on se contente d'y fixer un sac de façon à retenir les grains de caillé qui peuvent être entraînés par le.sérum "), (1). Avant d'être refoulé vers la porcherie voisine, le sérum s'écoule dans un bassin en ciment, revêtu d'une feuille de cuivre de $1 \mathrm{~mm}$ d'épaisseur (c). Quand " on le retourne " aux sociétaires, il est recueilli dans un bac spécial.

(a) Notre travail n'a trait qu'au "sérum à la présure ", le "sérum lactique, qui diffère beaucoup du premier par sa composition, ne nous arrêtera pas.

(b) Elles écrèment envíron 3.000 litres par heure et tournent à 6.000 tours par minute.

(c) Souvent le revêtement en cuivre n'existe pas, l'action corrosive du sérum sur l'enduit cimenté est alors intense. La formation de lactate de chaux détruit l'enduit en quelques semaines. 
Pour la caséine lactique, le lait écrémé, abandonné à lui-même a près addition d'une légère quantité de sérum de la veille ou levain, se coagule en moins de 24 heures et subit ensuite la même ouisson et préparation que la caséine à la présure. Ses débouchés sont plus réduits ; la production du sérum lactique est peu intense.

Propriétés physiques et chimiques. - Le sérum des beurreries-caséineries est un liquide jaunâtre, "couleur variable avec la nature du bétail " (2), louche, à cause des parcelles de caséine en suspension qui ne se déposent que peu à peu. L'odeur et la sa veur sont aigrelettes, surtout en été quand la fermentation lactique est intense. Sa chaleur spécifique est égale, à $15^{\circ}$, à 0.976 . Sa densité est de 1025 à 1030 .

Sa réaction est acide. Cette acidité, due au début, non à l'acide lactique mais aux seuls composants du sérum : phosphates, les acides en particulier, acide carbonique libre, etc... (2), se rapproche de 10 à $15^{\circ}$ Dornic (a). Sous l'influence de la température, l'acidité s'élève et pourrait, d'après BEAU, atteindre $120^{\circ}$ Dornic : fait assez rare dans la pratique où le chauffage à $63^{\circ}$, tuant les bacilles lactiques ou amoindrissant leur vitalité, empêche une fermentation ultérieure aussi poussée. L'acide lactique se forme au détriment du sucre; il peut être droit, gauche ou inactif, selon les ferments qui ont présidé à sa formation et la température à laquelle ils ont agi (3).

Dans cette fermentation, à $15-20^{\circ} \mathrm{C}$, il se formera uniquement de l'acide lactique; à une température plus élevée, apparaissent l'acide acétique et l'acide succinique et parfois l'alcool.

Les éléments du sérum sont : Des matières albuminoïdes, des sels et du sucre ainsi qu'il ressort du tableau suivant :

\begin{tabular}{|c|c|c|c|c|}
\hline & & Konig & Dornic-Daire & Berry (4) \\
\hline ... & . . . & 93,38 & 93 & 93,15 \\
\hline Albuminoïdes . . . & . . . , & 0,86 & 0,5 à 1 & 0,87 \\
\hline Sucre...... & . & 4,79 & 5,2 & 4,78 \\
\hline Matières grasses ... . & . & 0,32 & - & 0,23 \\
\hline Iatières solides totales. & . . & - & - & 6,86 \\
\hline
\end{tabular}

BERRY a montré, par des analyses faites d'avril à août, des variations saisonnières en sucre et en protéine, dont le maximum s'observe en juin et le minimum en avril (5).

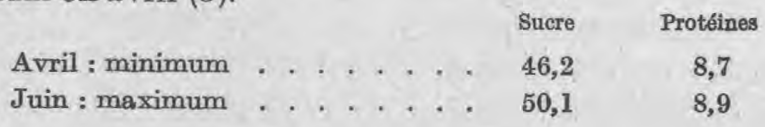

" En plus des variations de composition en sucre et en protéines, il y a de périodiques changements, de caractère subtil, qui ont un profond effet dans la condensation du sérum. Ces variations, qui a vaient été men-

(a) Le degré Dornic correspond à 0 gr. 10 d'acide lactique par litre. 
tionnées pour la condensation du lait, ont été attribuées à une action bactérienne: Elles relèvent de l'accroissement de calcium, due à la nature des aliments ou au vêlage (2). ,

" Le sérum contient 6 à $7 \%$ de cendres qu'il n'est pas possible de considérer comme le reflet des éléments salins originels du lait : La calcination est une opération brutale qui brise violemment, sans laisser la moindre trace de Ieur état antérieur, certaines liaisons existant entre différents éléments " (6).

Le sérum est beaucoup moins riche en phosphore que le lait; car, au moment de la coagulation de la caséine (phosphoprotéine) sous l'influence de la présure, se précipite un phospho-caséinate de chaux, complexe dans lequel, par adsorption, par teinture, le phosphate calcique et magnésien est resté étroitement uni au caséinate de chaux.

Les phosphates dissous sont des phosphates de potassium

environ $\begin{cases}\text { monopotassiques } & 1 \text { gramme } \\ \text { dipotassiques } & 1 \text { gramme } 10 .\end{cases}$

Le sérum est plus pauvre en phosphates inorganiques que le lait; $40 \%$ seulement (7). Les phosphates organiques manqueraient (8) ainsi que les lécithines insolubles, qui faisaient partie de l'enveloppe des globules gras (9) et les accompagnent dans la crème et le babeurre (10).

Le sérum renferme aussi les sels suivants :

Chlorure de sodium. . . . . . . . . . 0.0 gr. 960 à 1 gr. 090
Chlorure de potassium . . . . . . . . . . . . . 0 gr. 830 à 0 gr. 920
Sulfate de potassium 180

Les sels potassiques l'emportent pondéralement sur les sels calciques.

Il existe une relation étroite entre la teneur du lait en lactose et sa teneur en chlorure de sodium (11).

Les citrates (l'acide citrique dans le règne animal est une des caractéristiques de la sécrśtion mammaire) existent sous forme de :

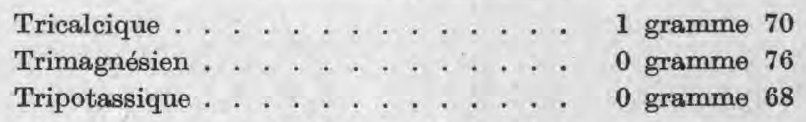

dont "l'ensemble constitue l'édifice salin homogène, le plus important du lait, puisqu'il dépasse 3 grammes, ce qui correspond à peu près au tiers. de toutes les matières salines " (6).

$26,6 \%$ du calcium seraient sous forme de citrate (12); il n'y a par contre pas de citrate de sodium.

De faibles quantités de fluore, d'iode, d'arsenic, de silicium, de bore, de lithium, de cuivre peuvent y être décelées (1). La teneur en fer serait moindre que celle indiquée par BUNGE (3), en 1884, soit 0 gr. 002.

Le sérum contient un sucre bien défini que la cellule mammairefabrique à partir du glucose, formé par le foie. C'est le même dans toutes: les espèces animales $(13)$ : le lactose. 
Le sérum de lait est pauvre en matières albuminoïdes, 5 à 10 grammes, représentées par la lactalbumine et par une faible quantité de caséine coagulée. Après action de la présure, il ne reste plus, en effet, contrairement à ce qu'affirmaient Hammartsen, Arthur, Pagès (3) la partie soluble de caséine $\left(1 / 10^{\circ}\right)$. La lactoglobuline, toujours si peu abondante, suit les globules gras dans la centrifugation. L'azote du sérum se répartit aussi entre des albumoses, des peptones, acide urique, urée, créatine, créatinine, dont les quantités ont été récemment calculées par BLEYER et KaLLMANN (14).

Les quantités d'azote varient, quelque peu, selon que les analyses portent sur du sérum à la présure ou du sérum lactique.

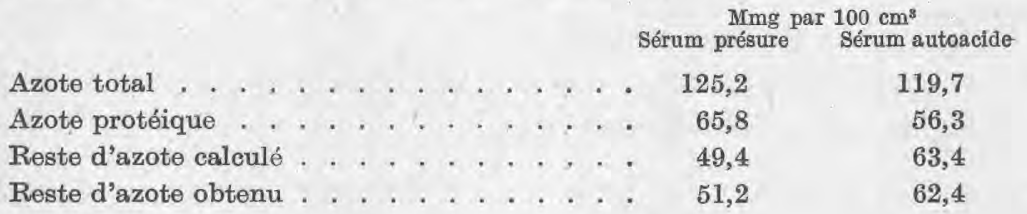

La lactalbumine, d'un rendement moyen de 3 gr. 20 par litre (12), et la plus complète, pour les moindres taux, de toutes les albumines, serait prépondérante. "Elle affecte un état colloïdal dit "moléculaire ". "Ses solutions, optiquement stables, n'ont aucune tendance à floculer. ». (1.5).

Sa composition n'est que partiellement connue : $62,10 \%$ de la molécule ont pu être analysés, l'autre portion échappe encore à toute analyse. Insoluble ou presque dans l'oxalate de sodium à $1 \%(16)$, elle renferme, d'après SÉBEILIEN, $1,75 \%$ de soufre et pas de phosphore (17). Sa coagulation commence à $67-70^{\circ}$ mais n'est compiète qu'à $76-78^{\circ}$.

La répartition de son azote entre les principaux groupes de corps. azotés est la suivante : (17)

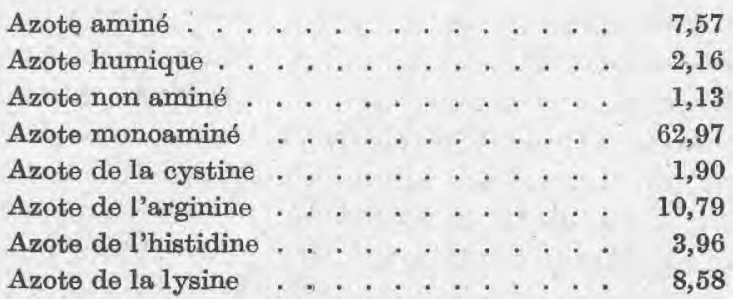

La lactalbumine abonde en acides-aminés, ainsi que l'indique le tableau suivant, emprunté à Purmmer (18) :

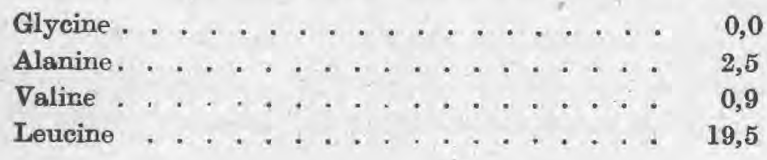




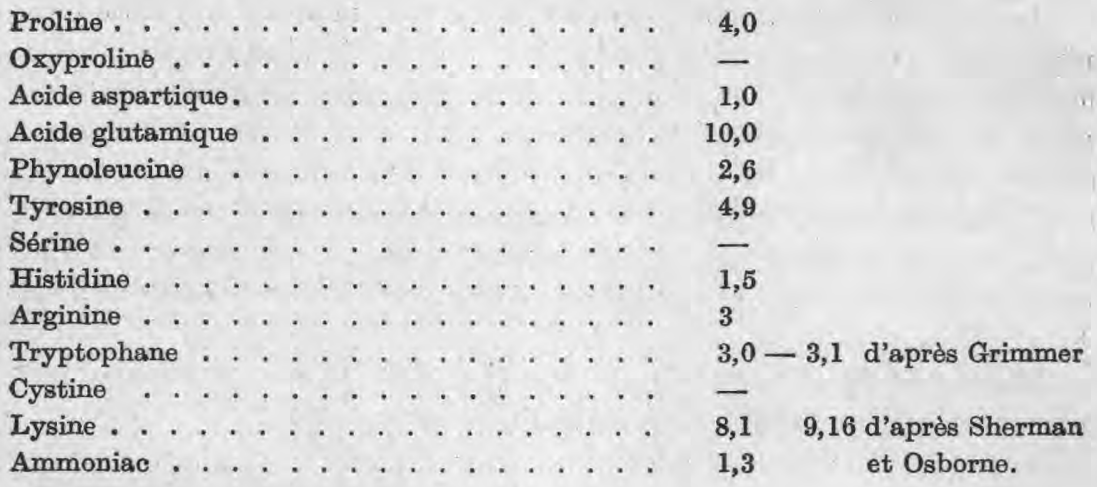

Le sérum, surtout le lactique, posséderait en pius des matières pondérabies, des diastases ou ferments solubles.

Valeur alimentaire. - Le sérum apporte done, en quantités considérables, des matières salines dont l'importance est manifeste. Malgré nos connaissances encore rudimentaires sur les besoins qualitatifs de l'organisme en matières minérales, nous n'ignorons cependant pas que celles-ci interviennent comme accélérateurs ou freinateurs dans tous les phénomènes, liés à la vie des cellules. Les unes ont un rôle essentiellement plastique; d'autres agissent à dose minime et semblent jouer le rôle d'éléments catalyseurs : "Ce ne sont souvent que des excitants du métabolisme " (19). OsBorne et Mendel n'ont-ils pas constaté que des régimes, dont les éléments contiennent très peu de chlore, sodium, magnésium ou potassium, permettent la croissance, tandis que le défaut simultané de sodium et de potassium la rend impossible ? La loi du minimum semble s'appliquer à certains sels comme elle s'applique, ainsi que nous le verrons plus tard, uux aminoacides. Enapportant, sous forme de composés organiques très assimilables, chlore, sodium et calcium, que les céréales ne fournissent qu'en quantités insuffisantes (20), le sérum corrigera les déficiences de la ration. De plus, la richesse en sels potassiques, qui l'emportent pondéralement, même sur les calciques, fera dépasser le minimum de $0,1 \%$, reconnu indispensable par MrLLeR (21). Le porc trouvera, dans les 15-20 litres, distribués quotidiennement en CharenteInférieure, la majeure partie du phosphore (22) et de la chaux qui lui sont nécessaires. Ce besoin, d'après OrR et HusBand (23) ne dépasse pas 3 à 5 grammes pour la chaux.

La pauvreté en fer est regrettable, sar CRICHTON et MAC Gowan ont montré que le jeune porcelet a besoin d'une forte accumulation de ce métal, dans son organisme en voie de croissance. Le manque de fer causerait, chez cet animal, de véritables anémies enzootiques (24).

Le sérum est riche en lactose. Les recherches d'ALBERTONI ont montré 
que, parmi les sucres solubles, il était un de ceux qui s'absorbent le plus facilement; bien que non directement assimilable (DASTRE), il n'est pas douteux qu'il ne se transforme en glycose en traversant l'organisme (25) (expérience de Bourquelot et Troissier).

Sa "dépense d'exploitation ", à peu près nulle tant que la ration n'est pas largement surabondante, est réduite au minimum puisqu'elle ne nécessite aucune mastication.

L'acide lactique du sérum, stade probablement obligatoire de l'utilisation des sucres (25), se comporte comme le glucose (Kocher).

Nous savons, depuis les études de VoIt, Korknerow, RuBnER, Luthje, Cathgart, qu'au cours de la croissance, "le besoin azoté peut être couvert par des quantités plus faibles d'albumine quand la ration contient comme aliments ternaires des hydrates de carbone, au moins à un certain taux, que lorsqu'elle renferme uniquement des matières grasses $)(27)$.

Aussi, lorsque le sérum est ajouté à une alimentation très azotée, des tourteaux par exemple, le lactose agira-t-il activement à la fois sur la rétention azotée et sur le métabolisme des matières grasses. Il fournira surtout, à l'organisme du porc, toutes les calories nécessaires (a), permettra la transformation intégrale en graisse des hydrates de carbone de la ration complémentaire, transformation que, dans certaines circonstances, il subira lui-même.

Les analyses nous ont montré une notable proportion d'acides-aminés. Ce fait est des plus intéressants. Les travaux de l'école biochimique américaine, et notamment ceux d'OsBorne et MENDEL, ont mis en évidence que le problème de la nutrition n'est pas résolu quand un régime correspond à un nombre de calories suffisant, fourni par des substances albuminoïdes quelconques, des graisses, des hydrates de carbone. "En plus de l'équilibre qui doit régner entre les divers principes, la ration exige des divers aliments certaines qualités " (27). Contrairement à ce que l'on admettait jusqu'ici, le minimum d'azote, nécessaire à la croissance ou à l'entretien, ne peut être emprunté indifféremment à toute substance protéique. "Les besoins de l'organisme ne peuvent être précisés que suivant un détail qualitatif très compliqué n (28).

" Au point de vue de leur rôle d'aliments plastiques, toutes les albumines n'ont pas la même valeur nutritive, évaluée d'après leur teneur en azote; cette différence est due au nombre, au taux, et surtout à la nature de leurs acides-aminés constitutifs ».

Les acides aminés, produits de la dislocation de la molécule pro. téique, se regroupent dans l'organisme pour constituer une albumine

(a) Ce besoin de calories varie aveo la surface du corps de l'animal, aveo la température extérieure. Il est proportiennellement moindre à mesure que l'animal grandit, le rapport entre la surface cutanée et le poids diminuant (26). 
spécifique. Or, l'organisme est incapable de créer "de toutes pièces certaines chaînes d'amino-acides (arginine, lysine) ou de former certains noyaux cycliques (tryptophane, etc.). Cette fonction de Cyclopoièse (Osboran et Mendec) est en dehors de ses moyens de synthèse " (30). Il doit emprunter ces amino-acides aux albumines étrangères et les $\mathrm{y}$ trouver en proportions optimum. On conçoit que ce travail de reconstruction se fera avec d'autant plus d'économie que l'albumine consommée se rapproshera davantage, par la nature et le mode d'association de ces amino-acides, de l'albumine spécifique à reconstruire. La nécessité, dans le métabolisme animal, d'acides-aminés contenant un noyau aromatique ou hétérocyclique et celle de certains acides diaminés est impérieuse. Si la valeur d'une albumine est réglée par la nature et le taux de ces amino-acides, sa valeur d'utilisation est en réalité réglée par le taux du fragment, le moins abondamment représenté. Cette loi a été établie par Osborne et Mender sous le nom de "Loi du Minimum » (28).

(A suivre.)

\title{
LA TENEUR EN MATIËRE GRASSE DU BABEURRE ET LA DURÉE DU BARATTAGE PEUVENT-ELLES ÊTRE INFLUENCÉES PAR LE GENRE DE LEVAIN LACTIQUE?
}

\author{
par W. VAN DAM et B. J. HOLWERDA.
}

Cette question nous fut posée par un praticien, le Chef technique de la Fédération des fabriques coopératives des produits de laiterie de la Frise, pau de temps après le début de nos expériences concernant cette question, expériences dont les résultats sont donnés dans cet article.

Nous avons essayé 17 levains d'origines différentes ; la plupart provenaient de fabriques de produits de laiterie de la Hollande du Nord, de la Frise, de l'Ovérijssel et de la Gueldre. Nous ne pouvions, à notre grand regret, à cause de la grande distance, espérer recevoir des levains en bon état du Limbourg et du Brabant. En dehors de quatre levains provenant de Hoorn et d'un cinquième qui, environ dix ans auparavant, avait été prélevé dans une fabrique de Giekerk, les douze autres levains provenaient tous de localités différentes. 35 expériences furent faites avec ces 17 levains ; chaque jour, deux ou trois levains furent comparés avec le levain utilisé à la fabrique d'expériences. Nous étions d'avis que cette comparaison était nécessaire, car on pouvait prévoir la possibilité d'anomalies dans une ou plusieurs cultures, provoquées par l'incubation dans un lait différent de celui auquel elles étaient habituées, et la comparaison avec la culture utilisée régulièrement à Hoorn pouvait constituer un moyen de contrôle plus ou moins efficace. La méthode - pératoire fut celle des expériences décrites dans les articles précédents. 